\title{
Intelligent Agriculture Mechanism using Internet of Things and Image Processing
}

\author{
Shubhada Bagal, Tejasvini More, Ankita Paranjape, Prof. Supriya Yadav
}

Department of Computer Engineering, NMIET, Talegaon Dabhade, Maharashtra, India

\begin{abstract}
This project presents a novel idea of detection and classification of leaf diseases and soil Moisture Level. It is difficult for human eyes to identify the exact type of leaf disease which occurs on the leaf of plant. Thus, in order to identify the leaf diseases accurately, the use of image processing and machine learning techniques can be helpful. The images used for this work were acquired from the field using digital camera. In pre-processing step, background removal technique is applied on the image in order to remove background from the image. Then, the background removed images are further processed for image segmentation using Otsu thresholding technique. Different segmented images will be used for extracting the features such as color, shape and texture from the images. At last, these extracted features will be used as inputs of classifier. Leaf diseases cause significant damage and economic losses in crops. Subsequently, reduction in leaf diseases by early diagnosis results in substantial improvement in quality of the product. Erroneous diagnosis of disease and its severity leads to inappropriate use of pesticides. The goal of proposed work is to diagnose the disease using image processing of plant leaf. In the proposed system, leaf image with complex background is taken as input. Thresholding is deployed to maskgreen pixels and image is processed to remove noise using anisotropic diffusion. Then leaf disease segmentation is done. The diseased portion from segmented images is identified. The purpose of this project is to developed our irrigation system, to make it automated and smart irrigation system. There are many countries where economy is depends on agriculture and the climatic conditions lead to lack of rains. The farmers working in the farm lands are dependent on the rains and bore wells. Even if the farm land has a water-pump, manual involvement by farmers is required to turn the pump on/off when on earth needed. The main purpose of this project is to measuring the moisture of agricultural soils by real-time method and to minimize this manual involvement by the farmer, which is why we are using a micro-controller. The sensor senses the amount of moisture present in the soil and presents an output in the form of resistance. If resistance is high then water is present in soil. Also we used another sensor to measure water level of well.
\end{abstract}

Keywords: Image Processing, Internet of Things, Sensors

\section{INTRODUCTION}

When we read the title of project first question comes in our mind that is what is perfect meaning of irrigation so the irrigation is the artificial application of water to the land or soil. it is used to assist in the crops, maintenance of landscapes and revegetation of disturbed soils in dry areas and during periods of inadequate rainfall. Irrigation comes from the Latin for"moist" or "wet" but it means the purposeful wetting of something. At the present era, the farmers have been using irrigation technique in India through the manual control in which the farmers irrigate the land at the regular intervals. The global irrigation scenario, however, is characterized by poor performance, increased demand for higher agricultural productivity, decreased availability of water for agriculture, increasing soil salinity and possible effects of global warming and climate change. This process sometimes consumes more water or sometimes the water reaches late due to which the crops get dried. Water deficiency can be detrimental to plants before visible wilting occurs. Slowed growth rate, lighter weight fruit follows slight water deficiency. This problem can be perfectly rectified if we use automatic microcontroller based rain gun irrigation system in which the irrigation will take place only when there will be intense requirement of water. Agriculture is a major source for majority of Indians and has great impact on the economy of the country. In dry areas or areas with inadequate rainfall, irrigation becomes a challenge. This technology helps in automation for proper yield and is handled remotely for farmer's safety. Hike in energy costs and decrease in water supplies points out the need for optimal water management. Irrigated water management involves more things than just turning on the irrigation system. Modern agriculture provides a range of benefits, including high yields and more income for farmers including small producers in both developed and developing countries. Irrigation management is a complex decisionmaking process to determine when and how much water is needed for growing crop as per specification of agricultural land. India is fast developing country and agriculture is the back bone for the countries development in the early stages. Due to industrialization and globalization concepts the field is facing hurdles. On top of that the awareness and the necessity of the cultivation need to be instilled in the minds of the younger generation. Now a day's technology plays vital role in all the fields but till today we are using some old methodologies in agriculture.

\section{MOTIVATION}

We get motivated by disadvantages of existing system.We have to match leaf images with trained database image using Scale invariant feature transform and detect specific diseases. In that system first we have pre-processing on that image then select feature extraction and compare leaf with database leaf and get the result by size calibration of the image for measuring diseases and Predict Soil Moisture.

\section{LITERATURE SURVEY}

1. Plant Disease Detection Using Leaf Pattern: Review(2015) 
Authors: Vishnu S, A. Ranjith Ram

Description: In this review paper we discuss the various methodologies for plant disease detection. Studies show that relying on pure naked-eye observation of experts to detect and classify diseases can be time consuming and expensive, especially in rural areas and developing countries. So we present fast, automatic, cheap and accurate image processing based solution. Solution is composed of four main phases; in the first phase we create a color transformation structure for the RGB leaf image and then, we apply color space transformation for the color transformation structure. Next, in the second phase, the images are segmented using the Kmeans clustering technique. In the third phase, we calculate the texture features for the segmented infected objects. Finally, in the fourth phase the extracted features are passed through a pre-trained neural network.

2. Paper name: Intelligent Agriculture mechanism using Internet of Things(2017)

Authors: Abhijith H V, Darpan A Jain, Adithya Athreya Rao U Description: In this paper we are using Intenet of things to make agriculture smarter. When IOT is implemented into the land, we establish a network of sensor nodes that are connected to each other. When the sensor nodes are deployed in the land, they will sense data at regular interval of time, perform the computations that are to be done and inform the farmer about the result of the computation. Internet of things is most promising and suitable technology for this.

3. Paper name: Detection of Diseases on Cotton Leaves Using K Mean Clustering Method(2015)

Authors: Pawan P. Warne, Dr. S. R. Ganorkar

Description: This paper presents an approach for careful detection of diseases, diagnosis and timely handling to prevent the crops from heavy losses. The diseases on the cotton are critical issue which makes the sharp decrease in the production of cotton. So for the study of interest is the leaf rather than whole cotton plant because about 85-95 percent of diseases occurred on the cotton leaves like Alternaria, Cercospora and Red Leaf Spot. In this proposal initially preprocessing the input image using histogram equalization is applied to increase the contrast in low contrast image, $\mathrm{K}$ means clustering algorithm is used for segmentation which classifies objects based on a set of features into $\mathrm{K}$ number of classes and finally classification is performed using Neural network. Thus image processing technique is used for detecting diseases on cotton leaves early and accurately. It is used to analyze the cotton diseases which will be useful to farmers

4. Paper name: Combining Local and Global Image Features for Object Class Recognition(2009)

Authors: Dimitri A. Lisin, Marwan A. Mattar, Matthe w B.Blaschko

Description: Object recognition is a central problem in computer vision research. Most object recognition Systems have taken one of two approaches, using either global or Local features exclusively. This may be in part due to the difficulty of combining a single global feature vector with a set of local features in a suitable manner. In this paper, we show that combining local and global features is beneficial in an application where rough segmentations of objects are available. We present a method for classification with local features using non-parametric Density estimation. Subsequently, we present two methods for combining Local and Global features. The first uses a "stacking" ensemble technique, and the Second uses a hierarchical classification system. Results show the superior performance of these combined methods over the component classifiers, with a reduction of over 20rate on a challenging marine science application.

5. Paper name: A Study and Implementation of Active Contour Model For Feature Extraction: With Diseased Cotton Leaf as Example(2014)

Authors: P.R. Rothe* and R. V. Kshirsagar

Description: Feature extraction is a significant constituent of a pattern recognition system. It carries out two assignments: converting input parameter vector into a feature vector and or reducing its dimensionality. A distinct feature extraction algorithm makes the classification process more effectual and efficient. The allocation and recognition of cotton leaf diseases are of the major importance as they have a cogent and momentous impact on quality and production of cotton. In this work we present a snake based approach for the segmentation of images of diseased cotton leaves. We extract Hu's moments which can be used as shape descriptors for classification. A theory of two-dimensional moment invariants for planar geometric figures is also presented. Three diseases have been considered, namely Bacterial Blight.

6. Paper name: Classification of Cotton Leaf Spot Diseases Using Image Processing Edge Detection Techniques(2012)

Authors: P.Revathi, M.Hemalatha

Description: This Proposed Work exposes, a advance computing technology that has been developed to help the farmer to take superior decision about many aspects of crop development process. Suitable evaluation and diagnosis of crop disease in the field is very critical for the increased production. Foliar is the major important fungal disease of cotton and occurs in all growing Indian regions. In this work we express new technological strategies using mobile captured symptoms of cotton leaf spot images and categorize the diseases using HPCCDD Proposed Algorithm . The classifier is being trained to achieve intelligent farming, including early Identification of diseases in the groves, selective fungicide application, etc. This proposed work is based on Image RGB feature ranging techniques used to identify the diseases (using Ranging values) in which, the captured images are processed for enhancement first. Then color image segmentation is carried out to get target regions (disease spots). Next Homogenize techniques like Sobel Dissertation Perception And Study and Canny filter are used to Identify the edges ,these extracted edge features are used in classification to identify the disease spots. Finally,pest recommendation is given to the farmers to ensure their crop and reduce the yeildloss.

\section{EXISTING SYSTEM}

Existing system is manual process. In existing system farmer need to visit nearest Agricultural Research Center. Then agriculture research center identify the disease on grape leaf after that farmer get know the disease and solution on disease. This is very long process and time consuming as well. Farmer needs to visit agriculture center frequently.

\section{Disadvantages:}

$>$ It is time Consuming

$>$ Error-prone

$>$ Required more time.

$>$ Farmers need to visit agriculture research center frequently. 


\section{ARCHITECTURE OF PROPOSED SYSTEM}

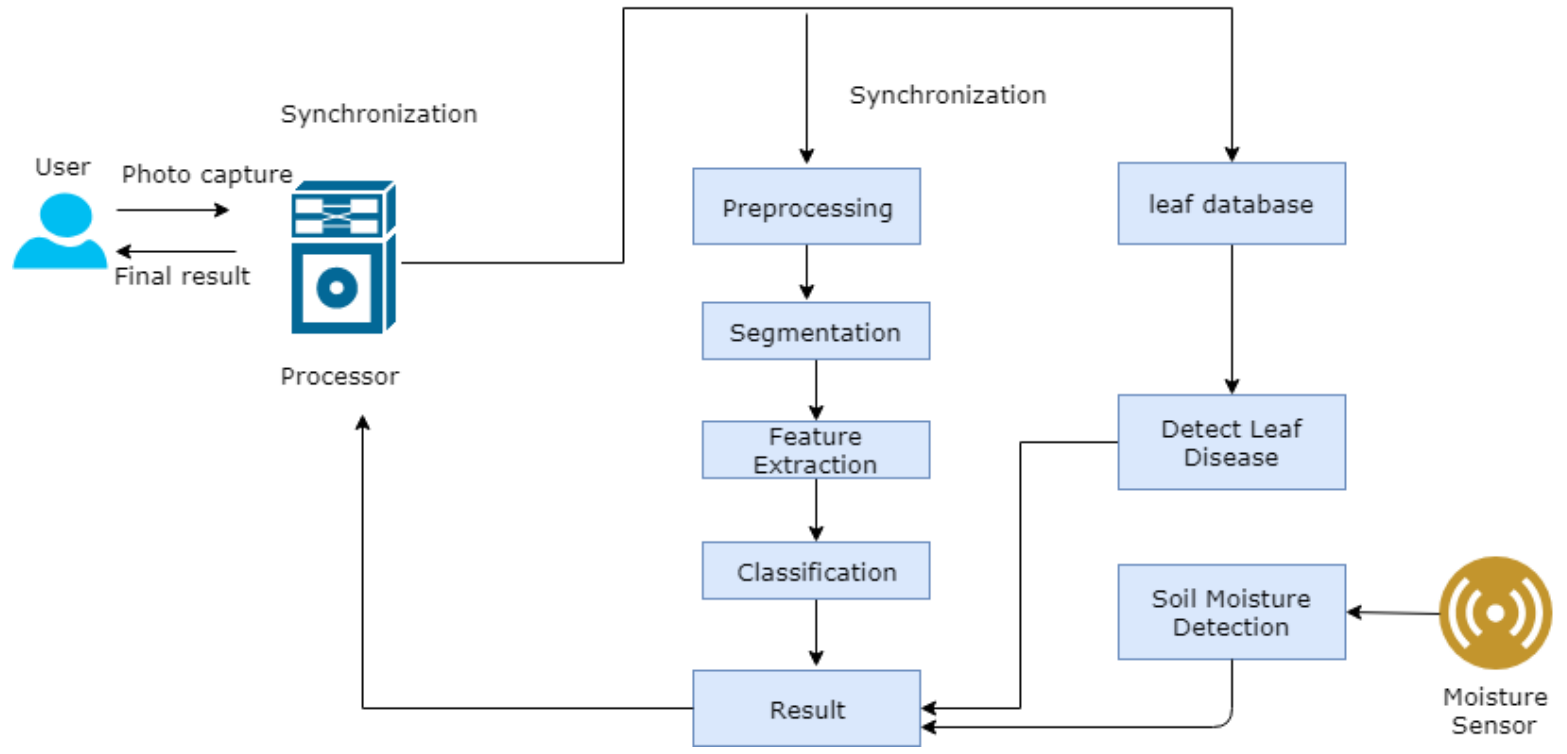

\section{PROPOSED SYSTEM}

In this project, we propose a mobile application that helps farmers for identifying leaf disease by uploading leaf image to the system. The system has an already trained dataset of images for the plant leaf. Input image given by the user undergoes several processing steps to detect the severity of disease by comparing with the trained database images. First the image is resized and then its features are extracted on parameters such as color. Next, classification is done to separate the image as infected or non-infected. And predict the moisture level in Soil and detect the water level.

\section{Advantages:}

$>$ Our system is built on leaf image processing and uses nutritional fact tables.

$>$ Required less time

$>$ The experimental result shows that our application is as accurate as a reference device.

$>$ We perform a detailed security analysis and performance evaluation of the proposed data

$>$ Increase Efficiency

$>$ Improve the accuracy.

VII. CONCLUSION

This project focuses on developing automated leaf diseases. It saves time and effort, In this project, we have proposed a new method for measuring the leaf diseases of the leaf object and find weather prediction. With then right combination of mobile and cloud computing we were able to obtain the estimated distance from the mobile device and used it to process the image in the cloud. This enabled us to process all the images from the same scale and further enabling us to determine the diseases value of the leaf object. Using deep learning we were able to extract the features of the leaf object and further classify it accurately. Study involved collecting leaf samples from different regions. Work was carried out to investigate the use of computer vision for classifying leaf diseases. Algorithms based on imageprocessing techniques, feature extraction and classification, were deployed. The feature extraction process used color cooccurrence methodology, which uses the texture of an image to arrive at unique features, which represent that image. Also by using Internet of things, Sensors and Data mining in Agriculture, we can help farmers to understand the timely requirements of the crop. With this, better yield can be generated. The usability of the proposed work is shown through the simulation. The action of deploying sensors in the agriculture land to sense the data and mining the data to identify the parameters required for better growth of crop is clearly shown through simulation. Prediction of specific diseases with respect to Sugarcane crop can be considered for future enhancement.

\section{REFERENCES}

[1] Les Baucum- "Sugarcane Plant Nutrient Diagnosis"

[2] "Package of Practices-Sugarcane" by Krishijagran.com (area under cultivation in India)

[3] Kwangho Jung1* and Sabinne Lee- "A systematic review of RFID applications and diffusion: key areas and public policy issues" ( RFID)

[4] Soong-hee Lee, DongIl Kim, Heechang Chung, Aekyung Moon, Song Li- "Ubiquitous Plant Farming based on networks " (ubiquitous plant farming)

[5] Shyamaladevi.K, T.T. Mirnalinee, Tina Esther Trueman , Kaladevi.R - "Design of Ontology Based Ubiquitous Web for Agriculture - A farmer helping system ."

[6] Ron Heiniger - "Sensors and Monitors for Measuring Soil Moisture"

[7] Junyan Ma, Xingshe Zhou, Shining Li, Zhigang Li "Connecting Agriculture to the Internet of Things through Sensor Networks" published in Internet of Things (iThings/CPSCom), 2011 International Conference on and 4th International Conference on Cyber, Physical and Social Computing.

[8] https://www.beechamresearch.com/files/BRLxecutive

[9] Wenjiang Huang, Qingsong Guan, Juhua Luo, Jingcheng Zhang, Jinling Zhao, Dong Liang, Linsheng Huang, and Dongyan Zhang, "New Optimized Spectral Indices for Identifying and Monitoring Winter Wheat Diseases", IEEE journal of selected topics in applied earth observation and remote sensing,Vol. 7, No. 6, June 2014

[10] Dr. K. Thangadurai, K. Padmavathi, "Computer Visionimage Enhancement For Plant Leaves Disease Detection", 2014 World Congress on Computing and Communication Technologies.

[11] Monica Jhuria, Ashwani Kumar, and Rushikesh Borse, "Image Processing For Smart Farming: Detection Of Disease And Fruit Grading", Proceedings of the 2013 IEEE Second International Conference on Image Information Processing (ICIIP-2013 